\section{Vaccine reactions}

To the editor-In your recent Vaccine Supplement, Burton and Moore discuss HIV vaccine development ${ }^{1}$. It is unfortunate that when discussing how best to proceed toward a vaccine, the debate is too often couched in the oversimplified terminology of empiric vaccine testing versus further basic research, as if the two were mutually exclusive. The process of HIV vaccine development will likely involve successive advances in understanding of immunopathogenesis along with experience gained by in vivo testing of candidate vaccines. Recent advances in the development of vaccines against rotavirus and malaria ${ }^{2}$ and improvements in established vaccines such as those against influenza and pertussis ${ }^{3}$, serve as examples of the beneficial interplay and mutual interdependence of basic immunology and animal and human efficacy testing.

Burton and Moore point out several properties of HIV-1 that may pose difficulties in eliciting an "effective" immune response. As an example, natural HIV-1 infection induces virus neutralizing antibodies only slowly over several years. In fact this "stealth-like" property of the envelope glycoprotein is also a characteristic of other animal lentiviruses ${ }^{4}$ and is perhaps not surprising for viruses that chronically replicate in their host. That said, we need to appreciate the limitations of our current understanding of what contributes to protection against HIV-1. The vast majority of HIV-1 infections are acquired via virus transmission across mucosal surfaces. The efficiency of transmission is low ${ }^{5}$ and little is known about the characteristics of local, regional and systemic immune responses that would provide protection against infection. Animal models used to evaluate potential vaccine candidates generally use high infectious doses of virus administered intravenously. These models may or may not predict efficacy in human HIV-1 transmission. Similarly, studies of vaccine immunogenicity often focus on neutralizing antibodies and cytotoxic T-lymphocytes. Although these are tangible and quantifiable in vitro measurements, they do not necessarily represent the entire repertoire of immune responses against HIV-1 nor do we know whether we are measuring them in a way that correlates with or predicts vaccineinduced protection.
Those of us committed to HIV-1 vaccine development must ensure that advances in understanding of HIV biology are expeditiously applied toward the science of HIV vaccine development. Similarly, clinical efficacy testing of vaccine candidates is necessary to develop and improve an effective vaccine. This bipartite strategy, far from sparking ideological debate, should foster cooperation and interdependence between scientists working on the basic and applied aspects of HIV vaccine development.

John R. Mascola, John G. MCNeil \& DEBORAH L. BIRX

Division of Retrovirology

Walter Reed Army Institute of Research

1 Taft Court, Suite 250

Rockville, MD 20580

email:jmascola@hiv.hjf.org

1. Burton, D.R. \& Moore, J.P. Why do we not have an HIV vaccine and how can we make one? Nature Med. 4, 495-498 (1998).

2. Miller, L.H. \& Hoffman, S.L. Research toward vaccines against malaria. Nature Med. 4, 520-524 (1998).

3. Rappuoli R. Rational design of vaccines. Nature Med. 3, 374-375 (1997).

4. Montelaro, R.C., Issel, C.J., Payne, S.L. \& Rushlow, K. Equine infectious anemia virus: A natural model for the immunologic management of lentivirus infections. In: AIDS vaccine research and clinical trials. (Putney, S.D. \& Bolognesi, D.P. eds.) pp 279-300 (Marcel Dekker, inc., New York, and Basel 1990).

5. Kunanusont, C. et al. HIV-1 subtypes and male-to-female transmission in Thailand. Lancet 345, 1078-1083 (1995).

To the editor-We are extremely concerned about your May issue news article "Are Japanese Researchers Exploiting Thai HIV Patients?". We believe it contains substantial misinformation and could seriously damage international scientific collaboration, delaying the development of urgently needed HIV vaccines-vaccines that are especially needed in developing countries. Therefore, we would like to clarify our situation with regard to JapanThai collaboration.

The joint Thai-Japanese project consists of research toward characterizing virus isolates from Thailand and preclinical development of HIV candidate vaccines. For one of the vaccine approaches (an approach using BCG-HIV recombinant vectors) we are collaborating with the Joint United Nations Programme on HIV/AIDS and the Global Programme on Vaccines and Immunization of the World Health Organization.

The HIV vaccine project will be conducted as a collaboration between researchers from both countries, building on a long-standing relationship between the Thai National Institute of Health and the Japanese National Institute of Infectious Diseases. Based on our preclinical experience using BCG-recombinant vectors for subtype B viruses, Japan will transfer technology to Thailand and explore with our Thai colleagues the development of candidate vaccines against HIV-1 subtype $E$, by far the most prevalent virus in Thailand.

Finally, once we have a promising candidate vaccine from these preclinical studies, it will be up to Thai scientists and authorities to decide whether or not to proceed to Phase I trials in Thailand, in accordance with the established scientific and ethical principles of international research. Since 1994, five Phase I/II HIV vaccine trials have been conducted in Thailand and we fully respect their excellent system for reviewing and approving proposals for such trials.

SHUDO YAMAZAKI

Director-General

National Institute of Infectious Diseases

1-23-1 Toyama

Shinjuku, Tokyo, Japan

yamazaki@nih.go.jp

To the editor-Burton and Moore claim that "The experience of the biopharmaceutical companies in the HIV vaccine area has not been a happy one ... . Interpretive loopholes are ruthlessly exploited to beathe life into a corpse ...." This is an opinion that we do not agree with. We do however agree that making an AIDS vaccine has been a challenge- a challenge made all the more difficult when well-meaning scientists overextend their expertise with language that polarizes the field. Biopharmaceutical companies, with their mandate to produce and test an HIV vaccine, and bench scientists, with their mandate to explore the base of academic knowledge, are not intrinsically at odds. If we wish to stop this epidemic as soon as possible, let everyone agree to stop sniping and instead focus on the problem before us.

DONALD P. Francis \& RoBERT C. NOWINSKI

VaxGen, 501 Forbes Boulevard

South San Francisco, CA 94080 USA 